\title{
DROUGHT STRESS INTENSITY, DURATION AND ITS RESISTANCE IMPACT ON RICE (ORYZA SATIVA L.) SEEDLING
}

\author{
CHEN, Z. K. ${ }^{1 \#}-$ XU, W. W. ${ }^{1 \#}-$ NIE, J. ${ }^{1}-$ KHAN, A. ${ }^{1}-$ CAO, C. G. ${ }^{1,2}-$ LI, P. ${ }^{1 *}$ \\ ${ }^{1}$ College of Plant Science and Technology, Huazhong Agricultural University, Wuhan, Hubei \\ 430070, China
}

${ }^{2}$ Hubei Collaborative Innovation Center for Grain Industry, Yangtze University, Jingzhou, Hubei 434025, China

${ }^{\#}$ These authors equally contributed to this work

*Corresponding author

e-mail:sleep1022@mail.hzau.edu.cn

(Received $7^{\text {th }}$ Sep 2019; accepted $15^{\text {th }}$ Nov 2019)

\begin{abstract}
Rice seedlings have a different response to drought stress intensity and duration, which reduces rice seedling growth and grain yield. In the year 2015, a greenhouse experiment was conducted to ascertain the effect of the relationship between drought stress intensity, duration, and drought resistance on rice seedling. Yangliangyou 6 ("super" rice variety) and Hanyou 113 (drought-resistance rice variety) were subjected to polyethylene glycol (PEG6000)-induced water stress. Drought stress intensities i.e. $10 \%, 15 \%, 20 \%$ and $25 \%$ PEG6000 with $4 \mathrm{~d}, 8 \mathrm{~d}$ or $12 \mathrm{~d}$ after emergence at 21 days was imposed, respectively. Control (0\% PEG6000) was used for comparison. Drought dehydration factor (DDF) and root induction factor (RIF) increased with drought duration. In each drought stress duration, RIF, drought tolerance factor (DTF) and DDF were raised under moderate drought stress intensity (10-15\% PEG6000), the advantage of DDF and DTF was shown at $12 \mathrm{~d}$ drought duration under severe drought stress intensity (20-25\% PEG6000). Thus, drought stress intensity significantly negatively affected rice seedling growth compared with drought stress duration. Improvement in DDF and RIF enhanced rice seedling growth under moderate drought stress conditions, while severe drought stress positively stimulated DDF and DTF which resulted in improved rice seedling growth.

Keywords: polyethylene glycol, drought tolerance, drought dehydration, root induction, dry mass
\end{abstract}

Abbreviations: ARI: adversity resistance index; CDRF: comprehensive drought resistant factor; CK: control treatment ( $0 \%$ polyethylene glycol 6000 solution); Co: conductivity; DDF: drought dehydration factor; DM: dry mass; DTF: drought tolerance factor; HY113: Hanyou 113; LA: leaf area; LRL: longest root length; MLL: mean leaf length; MLW: mean leaf width; PEG: polyethylene glycol; Pro: proline; RDM: root dry matter; RIF: root induction factor; RN: root number; RV: root volume; RWC: relative water content; SDM: shoot dry matter; SH: seedling height; SS: soluble sugar; TDM: total dry matter; YLY6: Yangliangyou 6

\section{Introduction}

Rice (Oryza sativa L.) is the most important staple food crop for about 33\% of people worldwide (Ye et al., 2013). Rice is grown under traditional flooding irrigation (Yang et al., 2008; Ye et al., 2013), but the shortage of water resources coupled with seasonal drought spell has limited rice production. Thus, stimulated the development of upland rice planting (Sun et al., 2012; Ye et al., 2013). Upland rice planting area has increased to 20 million ha worldwide and the planting area accounted for $12.7 \%$ of total rice planting area (Technology and Information Center of China Rice Research Institute, 2016). In China, due to limitations of hilly area, water shortage, suitable planting areas for upland rice were 5.3 million to 6.7 million ha (Technology and Information Center of China Rice Research 
Institute, 2016). But rice dry cultivation is still facing some challenges such as, variety selection, soil moisture conditions required for sowing and maintaining seedling growth. To overcome these challenges, it is important to improve planting technologies of upland rice to achieve optimistic resource utilization.

In current agricultural production systems, super rice variety (rice yield potential was about 10.5-12.0 $\mathrm{t} \mathrm{ha}^{-1}$ and increased by 8-15\% compared with ordinary hybrid and inbred varieties. This increment in yield was associated with more number per unit land area and large panicle size (Huang et al., 2011). Drought tolerance rice variety (the yield potential and good quality could be maintained under water-limited environments, as well as the capacity of water-saving or drought resistance (Luo et al., 2010)) were used in upland rice system to increase economic benefits. Upland rice is directly seeded by less water application which improved germination and emergence of rice seeds, and followed by no water application leading to seedling damage or death and consequently yields loss (Xu et al., 2017). Hence, it is necessary to define what modes of drought stress (different drought stress intensity and duration) had less effect on rice seedling during seedling stage. Also to identify drought tolerance of rice seedling growth in upland rice system. Rice dry cultivation mainly caused drought stress which impacted on rice seedling growth. Drought stress can induce various changes in morphological, metabolic and/or physiological functions and severely restrict both elongation and expansion of rice seedling (Kusaka et al., 2005). Drought stress also produced the reactive oxygen species (ROS), which could damage plant by lipid peroxidation, protein degradation, DNA fragmentation and ultimately cell death (Zhang et al., 2015). With increasing drought stress, shoot growth, root expansion and physiological function of rice seedling decreased (Pirdashti et al., 2003; Zheng et al., 2016). Increasing drought stress duration can restrain shoot growth of rice seedling and reducing yield and yield components (Asai et al., 2009; Duan et al., 2017). This indicated that drought stress intensity and duration had a negative effect on rice seedling growth. However, the effect of drought stress intensity coupled with duration on the rice seedling growth during seedling stage (after reviving stage) is lacking. Plants can avoid drought stress through two major mechanisms including drought dehydration and drought tolerance under drought stress conditions (Liu et al., 2003; Yue et al., 2006). Drought dehydration via using morphological plasticity of crops raised the water content of plant; drought tolerance indicated that crops could tolerate water loss and maintain certain metabolic or physiological processes such as, osmotic adjustment and antioxidant ability (Bandurska et al., 2003; Nazarli et al., 2014). Drought dehydration plays a crucial role in long-term drought duration, while drought tolerance had an obvious response to drought stress intensity (Yue et al., 2006; Farooq et al., 2009; Li et al., 2012). However, the relationship among the drought dehydration, tolerance, drought stress intensity and duration impact on rice seedling growth are poorly known.

Polyethylene glycol (PEG) is composed of a large molecular size and nontoxic in nature, therefore categorized as osmopriming reagents, which lowers the water potential without penetrating into seeds upon soaking (Chen and Arora, 2011; Zheng et al., 2016). Osmotic stress with PEG has been proposed to improve seed germination, early seedling vigor, antioxidant enzymes activity and eventually leading to increase stress tolerance in many crop plants (Chen and Arora, 2011). Seedlings death occurred at 8-12 $d$ with water break after emergence under severe drought stress (Chen and Arora, 2011). Thus, in this study PEG was used to simulate drought stress in $12 \mathrm{~d}$ at water break after emergence (1) to investigate the morphology of shoot and root, anti-active substance 
and the accumulation in rice seedling under different drought stress intensity and duration; (2) to examine the relationship between drought stress intensity and duration as well as drought resistance impacted on rice seedling growth.

\section{Materials and methods}

\section{Experiment site and design}

A greenhouse experiment was conducted at Huazhong Agricultural University, Hubei Province, China (E114 ${ }^{\circ} 9^{\prime}$, N30 $29^{\prime}$ ') in the 2017 growing season. Two rice varieties were used, Hanyou 113 (HY113), having the ability of drought resistance and water-saving. It was bred by Shanghai Agricultural Biological Gene Center (Shanghai province, China); Yangliangyou 6 (YLY6, "super" hybrid rice), indica and two-line hybrid rice, which was bred by Lixiahe Regional Research of Agricultural Science (Jiangsu province, China). It is widely cultivated in the middle and lower reaches of Yangtze River. The initial seed moisture contents of HY113 and YLY6 were $10.8 \%$ and $11.0 \%$, respectively. Rice seeds were sterilized with $25 \%$ prochloraz for $10 \mathrm{~min}$. The seeds of YLY6 and HY113 were sown in culture solution ( $\mathrm{pH}$ 5.0) at room temperature. The culture solution was a modified rice nutrient solution (nutrient solution scheme was provided by Internal Rice Research Institute) with the compositions (Table 1). The nutrient solution was renewed after 4 days. Three-week-old seedlings were transplanted to plastic buckets containing $(250 \mathrm{~mL}$, diameter $10 \mathrm{~cm}$, height $15 \mathrm{~cm})$ the same nutrient solution and renewed time. The room temperature and humidity were $25-30{ }^{\circ} \mathrm{C}$ and $80 \%$, respectively. The natural light was used during the whole experiment.

Table 1. Conventional nutrient solution

\begin{tabular}{c|c|c}
\hline Chemical compound & Formula weight & Concentration $(\mathbf{g} / \mathbf{L})$ \\
\hline $\mathrm{NH}_{4} \mathrm{NO}_{3}$ & 80.04 & 22.8578 \\
$\mathrm{NaH}_{2} \mathrm{PO}_{4} \cdot 2 \mathrm{H}_{2} \mathrm{O}$ & 156.01 & 10.072 \\
$\mathrm{~K}_{2} \mathrm{SO}_{4}$ & 174.26 & 17.8284 \\
$\mathrm{CaCl}_{2}$ & 110.99 & 22.1558 \\
$\mathrm{MgCl}_{2} \cdot 6 \mathrm{H}_{2} \mathrm{O}$ & 203.30 & 66.9182 \\
$\mathrm{Na}_{2} \mathrm{SiO}_{3} \cdot 9 \mathrm{H}_{2} \mathrm{O}$ & 284.20 & 9.52 \\
$\mathrm{MnSO}_{4} \cdot \mathrm{H}_{2} \mathrm{O}$ & 169.01 & 0.769 \\
$\mathrm{Na}_{2} \mathrm{MoO}_{4} \cdot 2 \mathrm{H}_{2} \mathrm{O}$ & 241.95 & 0.0605 \\
$\mathrm{H}_{3} \mathrm{BO}_{3}$ & 61.83 & 0.572 \\
$\mathrm{ZnSO}_{4} \cdot 7 \mathrm{H}_{2} \mathrm{O}$ & 287.56 & 0.0288 \\
$\mathrm{CuSO}_{4} \cdot 5 \mathrm{H}_{2} \mathrm{O}$ & 249.68 & 0.02 \\
$\mathrm{C}_{6} \mathrm{H}_{7} \mathrm{O}_{8} \cdot \mathrm{H}_{2} \mathrm{O}$ & 210.14 & 14.875 \\
$\mathrm{FeSO}_{4} \cdot 7 \mathrm{H}_{2} \mathrm{O}$ & 278.02 & 4.9766 \\
$\mathrm{EDTA}^{-\mathrm{Na}_{2}} \cdot 2 \mathrm{H}_{2} \mathrm{O}$ & 372.24 & 6.665 \\
\hline
\end{tabular}

PEG-6000 solution was used to induce osmotic stress on rice seedling (Chen and Arora, 2011; Zheng et al., 2016). Thirty treatments in each of eight replications (eight pots per replication and each pot comprised of one rice seedling) were used as follows: two varieties Yangliangyou 6 (YLY 6) and Hanyou 113 (HY113); five PEG-6000 solutions, i.e. $0 \%, 10 \%, 15 \%, 20 \%$ or $25 \%$ PEG-6000 ( $\mathrm{kg} \mathrm{kg}^{-1}$ (water)), the osmotic 
potential in each solution respectively was maintained at $0 \mathrm{kPa},-10 \mathrm{kPa},-15 \mathrm{kPa},-20$ $\mathrm{kPa}$ and $-25 \mathrm{kPa}$, among these 0\% PEG 6000 solution was used as control (CK); three drought duration with each PEG-6000 solutions to rice seedling were $4 \mathrm{~d}, 8 \mathrm{~d}$ and $12 \mathrm{~d}$. In addition, 10\%, 15\% and 20-25\% PEG-6000 concentration were regarded as mild, moderate and severe drought stress, respectively; and drought duration with $4 \mathrm{~d}, 8 \mathrm{~d}$ and $12 \mathrm{~d}$ were respectively referred to short-term, medium-term and long-term drought stress. Rice seedlings were watered with $0 \%, 10 \%, 15 \%, 20 \%$ or $25 \%$ PEG-6000 concentration at 5 th days after seedlings transplanted to the plot.

\section{Measurements}

At $4 \mathrm{~d}, 8 \mathrm{~d}$ and $12 \mathrm{~d}$ after drought stress with two repetitions each treatment, seedlings were uprooted, washed, and placed into an insulated box to prevent deterioration. Rice seedlings, relative water content (RWC), soluble sugar (SS), proline content (Pro) and conductivity (Co) were assessed according to Basu et al. (2010).

\section{Seedling morphological characteristics, dry mass and relative water content}

Shoot length (SH), longest root length (LRL), mean leaf length (MLL) and mean leaf width (MLW) were measured with a ruler; leaf area (LA) were measured by LI-3100C (LI-COR Inc., Lincoln, NE, USA). Seedlings were dissected into roots and shoots to assess the fresh weight (FW), respectively.

The roots of each seedling were spread in a plastic tray contained deionized water and scanned using a flatbed scanner (300 dpi). Root images were analyzed using WinRhizo image analysis software (Regent Instruments, Quebec, Canada). The software was configured to measure root volume $\left(\mathrm{RV}, \mathrm{cm}^{3}\right)$ and root number $(\mathrm{RN})$.

After scanning the roots, the root and shoot of rice seedling were oven-dried at $80^{\circ} \mathrm{C}$ for $48 \mathrm{~h}$, and shoot dry mass (SDM) and root dry mass (RDM) was weighed to obtain dry mass (DM). Adversity resistance index (ARI) was calculated by the formula (Wang et al., 2007):

$$
\mathrm{ARI}=\left(\frac{\mathrm{DM}_{(\mathrm{T})}}{\mathrm{DM}_{(\mathrm{CK})}}\right) \times \mathrm{DM}_{(\mathrm{A})}
$$

where $\mathrm{DM}_{(\mathrm{T})}$ was the dry mass of each treatment except $\mathrm{CK}, \mathrm{DM}_{(\mathrm{CK})}$ was the dry mass in $\mathrm{CK}, \mathrm{DM}_{(\mathrm{A})}$ was the average value of dry mass in all treatments including $\mathrm{CK}$.

RWC was calculated using the formula (Basu et al., 2010):

$$
\mathrm{RWC}=\frac{\mathrm{FW}-\mathrm{DW}}{\mathrm{FW}}
$$

where FW and DM respectively indicated the fresh weight and the dry mass of organs in rice seedling.

\section{Soluble sugar, proline content and conductivity}

Soluble sugar (SS) content was determined by Anthrone colorimetry (Zhang and Qu, 2003). A $1.0 \mathrm{ml}$ aliquot of the supernatant of tissue extract (root or shoot) was added 1.5 $\mathrm{mL}$ distilled water, which was mixed with $1 \mathrm{ml}$ of $9 \%$ phenol method, then 
homogenized in $5 \mathrm{~mL}$ of concentrated sulfuric acid. The mixture was at $25^{\circ} \mathrm{C}$ for 30 min. The absorption of chromophore was determined at $485 \mathrm{~nm}$ (Tecan-infinite M200, Switzerland).

Free proline content (Pro) of rice shoot and root were assayed according to the method (Bates et al., 1973). The samples were homogenized in $5 \mathrm{~mL}$ of $3 \%$ sulfosalicylic acid and centrifuged at $6000 \mathrm{rpm}$ for $10 \mathrm{~min}$. The supernatant of $2 \mathrm{~mL}$ was heated with $2 \mathrm{~mL}$ of ninhydrin and $2 \mathrm{~mL}$ glacial acetic acid at $100^{\circ} \mathrm{C}$ for $1 \mathrm{~h}$. The reaction was further extracted with $4 \mathrm{~mL}$ of toluene by vigorously vortexed for $30 \mathrm{~s}$. The absorption of chromophore was determined at $520 \mathrm{~nm}$.

To determine conductivity $(\mathrm{Co})$, shoot or root materials $(0.5 \mathrm{~g})$ were washed with deionized water and placed in $20 \mathrm{ml}$ deionized water tubes. The electrical conductivity of the solution was measured after $1 \mathrm{~h}$ of shaking at room temperature. Then samples were heated for $20 \mathrm{~min}$ and Co was measured. The Co measurements were performed (Li et al., 2013).

\section{Statistical analysis}

Analysis of variance (ANOVA), principal components analysis, correlation analysis and path analysis were performed using SPSS version 16.0 (SPSS Inc., Chicago, IL, USA) software. Differences between treatments were considered significant at $P<0.01$ and $P<0.05$ according to least significant difference (LSD) tests. Grey relational analysis was performed using DPS 7.5 (Zhejiang University, Hangzhou, China) software, gray correlation coefficient and grey incidence degrees were determined by the software. The value of the resolution ratio was 0.5 . The figures were plotted using Sigma Plot software version 10.0 (Systat Software Inc., San Jose, CA, USA). Data represent means $\pm \mathrm{SD}$.

\section{Results}

\section{The change of rice seedling morphological and physiological traits under drought stress intensity}

The value of the SH and RN had significant $(P<0.01)$ difference in drought stress intensity (Table 2), and reducing with the increasing PEG concentration, ARI raised and decreased later with increasing PEG concentration (Table 3$)$. The high value $(P<0.05)$ of ARI for YLY6 were resulted in 15\% PEG concentration level, AIR for HY113 in $10 \%$ PEG concentration level was significantly $(P<0.05)$ higher compared with others PEG concentrations. Both varieties had lower $(P<0.05)$ SH, MLL and MLW value when exposed to 20-25\% PEG concentration. And SH, MLL and MLW showed no significant differences among $0 \%, 10 \%$ and $15 \%$ PEG concentration. While the RN and RV were resulted in higher values at $10 \%$ PEG concentration, while produced significantly lower values in 20-25\% PEG concentration compared with $0 \%, 10 \%$ and $15 \%$ PEG levels. For both varieties, SS and Pro had higher value when subjected to 2025\% PEG concentration than other PEG concentration.

\section{The vary of rice seedling morphological and physiological traits under drought stress processing duration}

The SH, MLL, MLW, RN and RV were obviously influenced by drought stress duration (Table 2). Under both varieties, these parameters raised with the increase of 
drought duration and greater $(P<0.05)$ value were resulted at $12 \mathrm{~d}$ drought duration compared with other drought duration (Table 4). While SH, RV and RN at $12 \mathrm{~d}$ maintained the maximum value $(P<0.05)$ compared to $4 \mathrm{~d}$ and $8 \mathrm{~d}$ drought duration. Pro content was significantly reduced during the 8-12 d drought duration; while SS at $12 \mathrm{~d}$ lowered $(P<0.05)$ than that in $4 \mathrm{~d}$ and $8 \mathrm{~d}$ drought duration.

\section{The change of dry matter, leaf area, longest root length, conductivity and relative water content}

Drought stress intensity and duration had significantly $(P<0.01)$ individual and interactive effects on the shoot and root dry mass and total dry mass (Table 2). For both varieties, the greater TDM (95.9-97.7) was exhibited at $12 \mathrm{~d}$ drought duration at $10 \%$ and 15\% PEG concentration (Fig. 1); 15\% PEG concentration induced greater SDM (70.1-77.3) during $12 \mathrm{~d}$ drought duration. A higher RDM (27.5-28.7) was formed during $10 \%$ PEG concentration at $12 \mathrm{~d}$ drought duration. A lower TDM, SDM and RDM (11.011.4) were produced under 20-25\% PEG concentration during each drought duration.
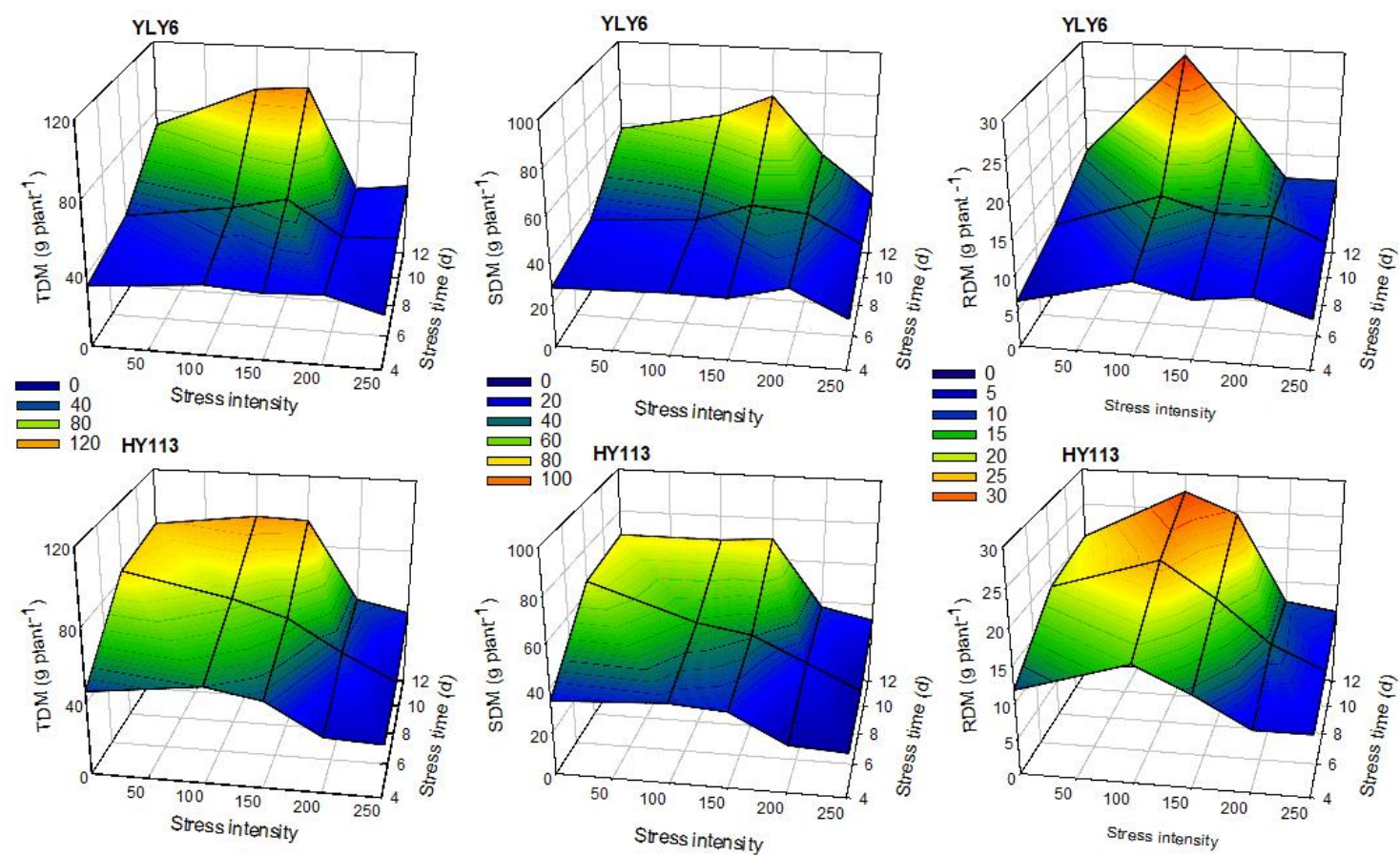

Figure 1. The total dry matter (TDM, $g$ plant $\left.{ }^{-1}\right)$, shoot dry matter (SDM, $g$ plant $\left.{ }^{-1}\right)$, root dry matter (RDM, $g$ plant $\left.^{-1}\right)$ in the coupling of drought stress intensity and stress duration under Yangliangyou 6 (YLY6) and Hanyou 113 (HY113)

The individual and interactive effects of drought stress intensity and duration significantly $(P<0.05)$ changed the LA and LRL. The greatest LA $(1.3-1.6)$ was observed for both varieties, respectively, at $12 \mathrm{~d}$ drought duration under $15 \%$ PEG level (Fig. 2). Both varieties had the greatest (10.7-13.1) LRL at $12 \mathrm{~d}$ processing duration with the 10\% PEG concentration, while lowest (5.1-8.2) value resulted from $20 \%$ and $25 \%$ PEG concentration. 

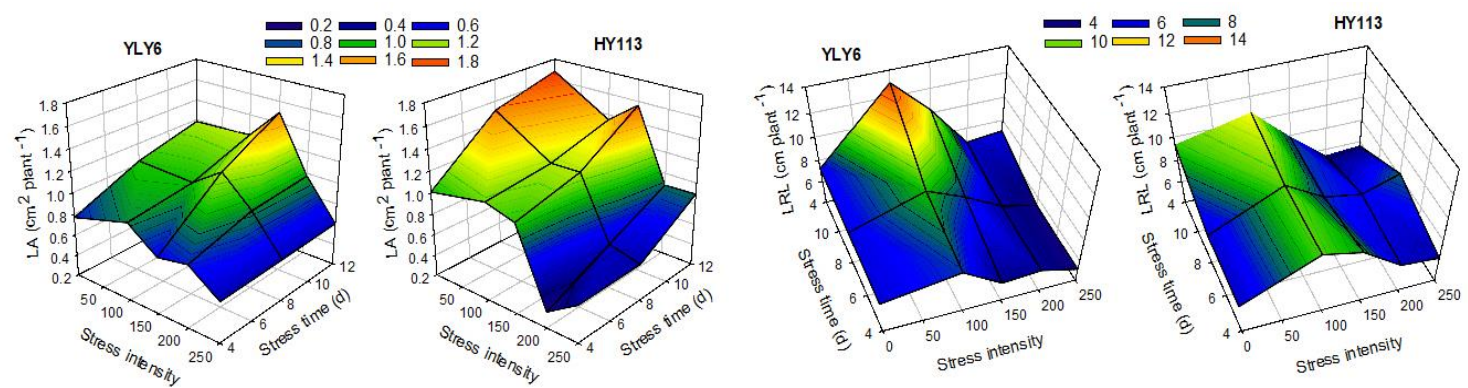

Figure 2. The leaf area $\left(L A, \mathrm{~cm}^{2}\right.$ plant $\left.^{-1}\right)$ and longest root length $\left(L R L, \mathrm{~cm}\right.$ plant $\left.{ }^{-1}\right)$ in the coupling of drought stress intensity and stress duration under Yangliangyou 6 (YLY6) and Hanyou 113 (HY113)

Drought stress intensity and duration had significant $(P<0.05)$ interactive effects on Co and RWC (Table 2). The Co showed peak (472.3) value at $8 \mathrm{~d}$ drought duration with 25\% PEG concentration (Fig. 3), while the valley (17.5-72.9) appeared at $12 \mathrm{~d}$ drought duration within $0-15 \%$ PEG concentration. A lower value of RWC (0.71-0.78) resulted at $20 \%$ and $25 \%$ PEG concentration during each processing duration compared with other PEG concentrations.
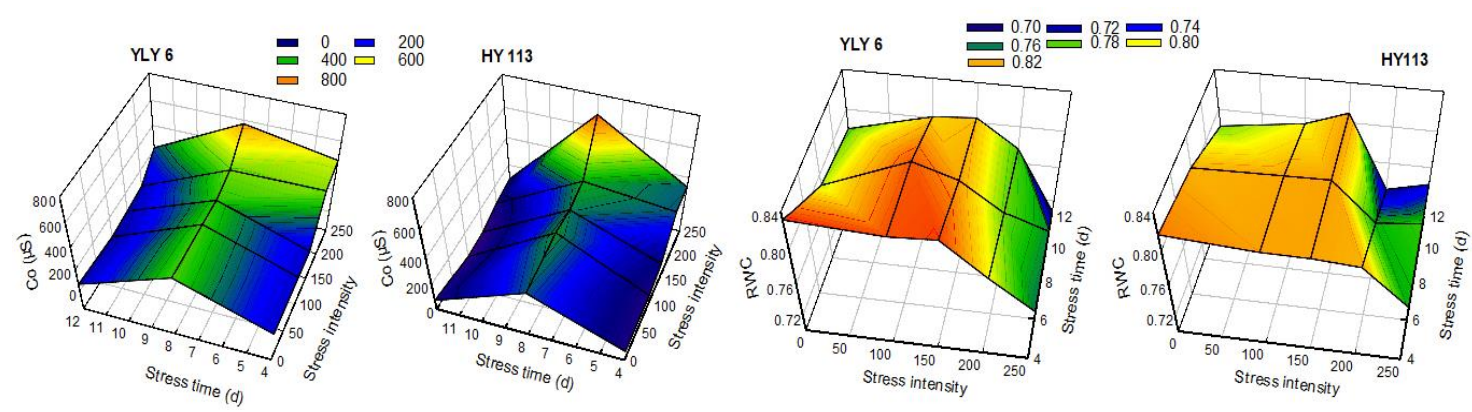

Figure 3. The conductivity $(C o, \mu S)$ and conductivity $(C o, \mu S)$ in the coupling of drought stress intensity and stress duration under Yangliangyou 6 (YLY6) and Hanyou 113 (HY113)

Table 2. The shoot dry matter (SDM, $g$ plant $\left.{ }^{-1}\right)$, root dry matter $\left(R D M, g\right.$ plant $\left.^{-1}\right)$, adversity resistance index $(A R I)$, relative water content $(R W C)$, seedling height $\left(S H, \mathrm{~cm}^{2}\right.$ plant $\left.{ }^{-1}\right)$, mean leaf length (MLL, cm plant $\left.{ }^{-1}\right)$, mean leaf width $\left(M L W, \mathrm{~cm}_{\text {plant }}{ }^{-1}\right)$, leaf area $\left(L A, \mathrm{~cm}^{2}\right.$ plant $\left.{ }^{-1}\right)$, longest root length ( LRL, $\left.\mathrm{cm}_{\text {plant }}{ }^{-1}\right)$, root number $\left(R N\right.$, plant $\left.{ }^{-1}\right)$, root volume $\left(R V, \mathrm{~cm}^{3}\right.$ plant $\left.{ }^{-1}\right)$, conductivity $(\mathrm{Co}, \mu \mathrm{S})$, soluble sugar (SS, \%), proline (Pro, $\mu \mathrm{g} \mathrm{g}^{-1}$ ) and total dry matter ( $g$ plant $\left.{ }^{1}\right)$ of drought stress intensity and stress duration under Yangliangyou 6 (YLY6) and Hanyou 113 (HY113)

\begin{tabular}{c|c|c|c|c|c|c|c|c|c|c|c|c|c|c|c}
\hline & SDM & RDM & ARI & RWC & SH & MLL & MLW & LA & LRL & RN & RV & Co & SS & Pro & TDM \\
\hline $\mathrm{SI}$ & $* *$ & $* *$ & $* *$ & $* *$ & $* *$ & $* *$ & $* *$ & $* *$ & $* *$ & $* *$ & $* *$ & $* *$ & $* *$ & $* *$ & $* *$ \\
$\mathrm{SD}$ & $* *$ & $* *$ & $\mathrm{~ns}$ & $* *$ & $* *$ & $* *$ & $* *$ & $* *$ & $* *$ & $* *$ & $* *$ & $* *$ & $* *$ & $* *$ & $* *$ \\
$\mathrm{~V}$ & $\mathrm{~ns}$ & $* *$ & $* *$ & $\mathrm{~ns}$ & $* *$ & $* *$ & $* *$ & $* *$ & $* *$ & $* *$ & $* *$ & $\mathrm{~ns}$ & $* *$ & $\mathrm{~ns}$ & $* *$ \\
$\mathrm{SI} \times \mathrm{SD}$ & $* *$ & $* *$ & $\mathrm{~ns}$ & $\mathrm{~ns}$ & $* *$ & $* *$ & $* *$ & $* *$ & $* *$ & $*$ & $* *$ & $* *$ & $* *$ & $* *$ & $* *$ \\
$\mathrm{SI} \times \mathrm{V}$ & $* *$ & $* *$ & $*$ & $\mathrm{~ns}$ & $* *$ & $* *$ & $* *$ & $* *$ & $* *$ & $\mathrm{~ns}$ & $* *$ & $\mathrm{~ns}$ & $* *$ & $\mathrm{~ns}$ & $* *$ \\
$\mathrm{SD} \times \mathrm{V}$ & $\mathrm{ns}$ & $\mathrm{ns}$ & $\mathrm{ns}$ & $\mathrm{ns}$ & $\mathrm{ns}$ & $* *$ & $*$ & $\mathrm{~ns}$ & $* *$ & $* *$ & $* *$ & $* *$ & $\mathrm{~ns}$ & $* *$ & $\mathrm{~ns}$ \\
$\mathrm{SI} \times \mathrm{SD} \times \mathrm{V}$ & $*$ & $\mathrm{~ns}$ & $\mathrm{~ns}$ & $\mathrm{~ns}$ & $*$ & $\mathrm{~ns}$ & $*$ & $\mathrm{~ns}$ & $* *$ & $* *$ & $* *$ & $* *$ & $* *$ & $* *$ & $\mathrm{~ns}$ \\
\hline
\end{tabular}

"SI" means drought stress intensity, "SD" denotes drought stress duration, and "V" indicates varieties. "ns" indicates nonsignificant. "**" and “**” mean significantly different at $P=0.05$ and $P=0.01$ according to Duncan's range test. The same below 
Table 3. The shoot dry matter (SDM, $g$ plant $\left.{ }^{-1}\right)$, root dry matter (RDM, $g$ plant $\left.{ }^{-1}\right)$, adversity resistance index (ARI), relative water content (RWC), seedling height $\left(S H, \mathrm{~cm}_{\text {plant }}{ }^{-1}\right)$, mean leaf length $\left(M L L, \mathrm{~cm} \mathrm{plant}^{-1}\right)$, mean leaf width $\left(M L W, \mathrm{~cm}_{\text {plant }}{ }^{-1}\right)$, leaf area $\left(L A, \mathrm{~cm}^{2}\right.$ plant $\left.{ }^{-1}\right)$, longest root length (LRL, cm plant $\left.{ }^{-1}\right)$, root number (RN, plant $\left.{ }^{-1}\right)$, root volume $\left(R V, \mathrm{~cm}^{3}\right.$ plant $\left.^{-1}\right)$, conductivity $(C o, \mu S)$, soluble sugar (SS, \%), proline (Pro, $\left.\mu g g^{-1}\right)$ and total dry matter $\left(\mathrm{g}\right.$ plant $\left.^{-1}\right)$ of drought stress intensity under Yangliangyou 6 (YLY6) and Hanyou 113 (HY113)

\begin{tabular}{|c|c|c|c|c|c|c|c|c|c|c|}
\hline \multirow{2}{*}{$\begin{array}{c}\text { Varieties } \\
\text { Stress intensity }(\%)\end{array}$} & \multicolumn{5}{|c|}{ YLY6 } & \multicolumn{5}{|c|}{ HY113 } \\
\hline & $\mathbf{0}$ & 10 & 15 & 20 & 25 & $\mathbf{0}$ & 10 & 15 & 20 & 25 \\
\hline $\operatorname{SDM}\left(\right.$ g plant $\left.^{-1}\right)$ & $39.51 \pm 1.70 \mathrm{c}$ & $44.33 \pm 1.81 \mathbf{b}$ & $50.59 \pm 2.15 \mathbf{a}$ & $42.84 \pm 1.81 \mathrm{~b}$ & $28.32 \pm 0.79 d$ & $56.01 \pm 2.04 \mathrm{a}$ & $51.45 \pm 1.60 \mathbf{a}$ & $50.00 \pm 1.68 \mathbf{a}$ & $30.86 \pm 1.36 \mathrm{~b}$ & $24.75 \pm 0.84 \mathrm{~b}$ \\
\hline RDM (g plant $\left.{ }^{-1}\right)$ & $9.82 \pm 0.49 b$ & $17.84 \pm 0.86 \mathbf{a}$ & $13.79 \pm 0.59 \mathbf{a b}$ & $11.13 \pm 0.86 b$ & $9.27 \pm 0.36 b$ & $16.96 \pm 0.46 b$ & $22.37 \pm 0.71 \mathbf{a}$ & $18.66 \pm 0.59 b$ & $11.12 \pm 0.44 \mathrm{c}$ & $9.66 \pm 0.32 c$ \\
\hline ARI & $0.92 \pm 0.02 b$ & $1.49 \pm 0.07 \mathbf{a}$ & $1.41 \pm 0.16 \mathbf{a}$ & $1.26 \pm 0.14 b$ & $0.62 \pm 0.07 \mathrm{c}$ & $1.25 \pm 0.06 \mathrm{a}$ & $1.23 \pm 0.06 \mathbf{a}$ & $1.08 \pm 0.08 \mathrm{~b}$ & $0.38 \pm 0.01 \mathrm{c}$ & $0.40 \pm 0.10 \mathrm{c}$ \\
\hline RWC & $0.80 \pm 0.00 \mathrm{a}$ & $0.82 \pm 0.01 \mathbf{a}$ & $0.81 \pm 0.00 \mathbf{a}$ & $0.78 \pm 0.00 \mathrm{a}$ & $0.74 \pm 0.01 \mathbf{b}$ & $0.80 \pm 0.01 \mathrm{a}$ & $0.80 \pm 0.00 \mathrm{a}$ & $0.81 \pm 0.01 \mathrm{a}$ & $0.77 \pm 0.01 \mathrm{a}$ & $0.76 \pm 0.01 \mathrm{a}$ \\
\hline $\mathrm{SH}\left(\mathrm{cm}\right.$ plant $\left.^{-1}\right)$ & $21.45 \pm 0.32 \mathrm{a}$ & $20.30 \pm 0.27 \mathbf{a b}$ & $20.83 \pm 0.37 \mathbf{a b}$ & $19.39 \pm 0.27 \mathrm{ab}$ & $18.21 \pm 0.08 \mathbf{b}$ & $22.21 \pm 0.37 \mathrm{a}$ & $20.00 \pm 0.26 \mathbf{b}$ & $19.62 \pm 0.16 \mathbf{b}$ & $15.71 \pm 0.22 \mathrm{c}$ & $16.08 \pm 0.14 \mathrm{c}$ \\
\hline $\operatorname{MLL}\left(\mathrm{cm} \mathrm{plant}^{-1}\right)$ & $9.70 \pm 0.18 \mathrm{a}$ & $10.04 \pm 0.02 \mathbf{a}$ & $10.37 \pm 0.08 \mathbf{a}$ & $10.53 \pm 0.02 \mathrm{a}$ & $8.63 \pm 0.09 \mathbf{b}$ & $11.18 \pm 0.18 \mathrm{a}$ & $10.33 \pm 0.07 \mathbf{a}$ & $10.27 \pm 0.10 \mathbf{a}$ & $7.34 \pm 0.08 b$ & $7.93 \pm 0.02 b$ \\
\hline MLW $\left(\mathrm{cm} \mathrm{plant}^{-1}\right)$ & $0.32 \pm 0.01 \mathrm{a}$ & $0.33 \pm 0.01 \mathbf{a}$ & $0.32 \pm 0.01 \mathbf{a}$ & $0.27 \pm 0.01 b$ & $0.21 \pm 0.01 \mathbf{c}$ & $0.39 \pm 0.01 \mathrm{a}$ & $0.37 \pm 0.00 \mathbf{a}$ & $0.38 \pm 0.01 \mathbf{a}$ & $0.26 \pm 0.01 b$ & $0.26 \pm 0.01 b$ \\
\hline LA $\left(\mathrm{cm}^{2}\right.$ plant $\left.^{-1}\right)$ & $0.99 \pm 0.03 \mathrm{a}$ & $1.05 \pm 0.02 \mathbf{a}$ & $1.07 \pm 0.03 \mathbf{a}$ & $0.90 \pm 0.02 \mathrm{a}$ & $0.59 \pm 0.01 \mathbf{b}$ & $1.40 \pm 0.03 \mathrm{a}$ & $1.22 \pm 0.02 \mathbf{a}$ & $1.26 \pm 0.03 \mathbf{a}$ & $0.62 \pm 0.03 b$ & $0.66 \pm 0.03 b$ \\
\hline $\operatorname{LRL}\left(\mathrm{cm}\right.$ plant $\left.{ }^{-1}\right)$ & $7.03 \pm 0.08 c$ & $10.00 \pm 0.26 \mathbf{a}$ & $7.92 \pm 0.23 b$ & $6.24 \pm 0.26 \mathrm{~d}$ & $5.80 \pm 0.14 \mathrm{~d}$ & $7.56 \pm 0.22 c$ & $9.93 \pm 0.19 \mathbf{a}$ & $8.11 \pm 0.17 b$ & $6.37 \pm 0.17 \mathrm{c}$ & $8.37 \pm 0.33 b$ \\
\hline $\mathrm{RN}\left(\right.$ plant $\left.^{-1}\right)$ & $17.33 \pm 0.17 \mathrm{a}$ & $15.72 \pm 0.40 \mathbf{a b}$ & $14.00 \pm 0.34 \mathrm{~b}$ & $14.44 \pm 0.4 \mathrm{ab}$ & $13.00 \pm 0.31 b$ & $19.33 \pm 0.70 \mathrm{a}$ & $16.78 \pm 0.24 \mathbf{b}$ & $16.78 \pm 0.33 \mathbf{b}$ & $14.56 \pm 0.53 \mathrm{c}$ & $13.22 \pm 0.27 \mathrm{c}$ \\
\hline $\mathrm{RV}\left(\mathrm{cm}^{3}\right.$ plant $\left.^{-1}\right)$ & $0.09 \pm 0.01 \mathrm{c}$ & $0.15 \pm 0.01 \mathbf{a}$ & $0.13 \pm 0.01 b$ & $0.09 \pm 0.01 \mathrm{c}$ & $0.07 \pm 0.00 \mathrm{~d}$ & $0.12 \pm 0.00 \mathrm{a}$ & $0.15 \pm 0.01 \mathbf{a}$ & $0.14 \pm 0.01 \mathbf{a}$ & $0.06 \pm 0.00 \mathrm{~b}$ & $0.07 \pm 0.00 \mathrm{~b}$ \\
\hline $\operatorname{Co}(\mu \mathrm{S})$ & $168.3 \pm 14.41 \mathrm{c}$ & $150.6 \pm 15.01 \mathrm{c}$ & $152.0 \pm 13.82 \mathrm{c}$ & $280.0 \pm 15.01 \mathrm{~b}$ & $376.8 \pm 13.42 \mathbf{a}$ & $159.1 \pm 13.07 \mathrm{c}$ & $154.7 \pm 16.71 \mathrm{c}$ & $167.9 \pm 15.17 \mathrm{c}$ & $262.5 \pm 15.80 \mathrm{~b}$ & $369.8 \pm 26.44 \mathbf{a}$ \\
\hline SS (\%) & $1.10 \pm 0.03 b$ & $1.33 \pm 0.04 b$ & $1.26 \pm 0.03 b$ & $1.47 \pm 0.04 \mathrm{~b}$ & $1.86 \pm 0.06 \mathbf{a}$ & $1.01 \pm 0.02 b$ & $1.28 \pm 0.03 b$ & $1.33 \pm 0.04 \mathrm{~b}$ & $1.74 \pm 0.04 \mathbf{a}$ & $1.94 \pm 0.05 \mathbf{a}$ \\
\hline $\operatorname{Pro}\left(\mu g^{-1}\right)$ & $2.23 \pm 0.12 c$ & $2.85 \pm 0.15 \mathrm{c}$ & $2.68 \pm 0.13 c$ & $4.61 \pm 0.15 b$ & $5.83 \pm 0.29 \mathbf{a}$ & $2.40 \pm 0.11 \mathrm{c}$ & $2.61 \pm 0.14 \mathrm{c}$ & $2.68 \pm 0.12 c$ & $4.19 \pm 0.20 b$ & $5.50 \pm 0.38 \mathrm{a}$ \\
\hline TDM $\left(\right.$ g plant $\left.^{-1}\right)$ & $49.33 \pm 2.14 b$ & $62.18 \pm 2.66 \mathbf{a}$ & $64.38 \pm 2.71 \mathbf{a}$ & $53.97 \pm 2.66 \mathrm{~b}$ & $37.59 \pm 1.12 \mathrm{c}$ & $72.97 \pm 2.41 \mathrm{a}$ & $73.82 \pm 2.26 \mathrm{a}$ & $68.66 \pm 2.21 \mathrm{a}$ & $41.98 \pm 1.78 b$ & $34.40 \pm 1.14 \mathrm{~b}$ \\
\hline
\end{tabular}

Bars indicate SD $(n=3)$. Different letters indicate a significant difference $(\mathrm{p}<0.05)$ between the stress intensity 
Table 4. The shoot dry matter (SDM, g plant $\left.{ }^{-1}\right)$, root dry matter (RDM, g plant $\left.{ }^{-1}\right)$, adversity resistance index (ARI), relative water content (RWC), seedling height ( $\left.\mathrm{SH}, \mathrm{cm}_{\text {plant }}^{-1}\right)$, mean leaf length $\left(M L L, \mathrm{~cm}_{\text {plant }}{ }^{-1}\right)$, mean leaf width $\left(M L W, \mathrm{~cm}\right.$ plant $\left.{ }^{-1}\right)$, leaf area $\left(L A, \mathrm{~cm}^{2}\right.$ plant $\left.{ }^{-1}\right)$, longest root length (LRL, cm plant $\left.{ }^{-1}\right)$, root number (RN, plant $\left.{ }^{-1}\right)$, root volume $\left(R V, \mathrm{~cm}^{3}\right.$ plant $\left.^{-1}\right)$, conductivity $(C o, \mu S)$, soluble sugar (SS, \%), proline (Pro, $\left.\mu g g^{-1}\right)$ and total dry matter $\left(\mathrm{g}\right.$ plant $\left.^{-1}\right)$ of drought stress duration under Yangliangyou 6 (YLY6) and Hanyou 113 (HY113)

\begin{tabular}{|c|c|c|c|c|c|c|}
\hline \multirow{2}{*}{$\begin{array}{c}\text { Varieties } \\
\text { Stress duration (d) }\end{array}$} & \multicolumn{3}{|c|}{ YLY6 } & \multicolumn{3}{|c|}{ HY113 } \\
\hline & 4 & 8 & 12 & 4 & 8 & 12 \\
\hline SDM $\left(\right.$ g plant $\left.^{-1}\right)$ & $28.87 \pm 4.74 b$ & $39.24 \pm 10.27 b$ & $54.24 \pm 19.85 \mathbf{a}$ & $29.85 \pm 8.50 \mathrm{c}$ & $42.69 \pm 19.67 \mathrm{~b}$ & $55.30 \pm 19.44 \mathbf{a}$ \\
\hline RDM (g plant $\left.{ }^{-1}\right)$ & $8.29 \pm 2.05 b$ & $12.15 \pm 3.38 b$ & $16.39 \pm 7.89 \mathbf{a}$ & $11.64 \pm 3.57 \mathrm{~b}$ & $16.52 \pm 6.65 \mathbf{a}$ & $19.09 \pm 7.86 \mathbf{a}$ \\
\hline ARI & $1.12 \pm 0.36 \mathrm{a}$ & $1.19 \pm 0.33 \mathrm{a}$ & $1.12 \pm 0.57 \mathrm{a}$ & $1.02 \pm 0.36 \mathrm{a}$ & $0.91 \pm 0.45 \mathrm{a}$ & $1.03 \pm 0.48 \mathrm{a}$ \\
\hline RWC & $0.81 \pm 0.04 \mathrm{a}$ & $0.80 \pm 0.04 \mathrm{a}$ & $0.77 \pm 0.05 \mathrm{a}$ & $0.80 \pm 0.05 a$ & $0.79 \pm 0.06 \mathrm{a}$ & $0.77 \pm 0.05 a$ \\
\hline SH (cm plant $\left.{ }^{-1}\right)$ & $18.77 \pm 1.56 \mathrm{~b}$ & $19.04 \pm 1.30 \mathrm{~b}$ & $22.09 \pm 3.31 \mathbf{a}$ & $17.38 \pm 2.09 b$ & $18.44 \pm 3.29 b$ & $20.35 \pm 4.10 \mathbf{a}$ \\
\hline $\operatorname{MLL}\left(\mathrm{cm} \mathrm{plant}^{-1}\right)$ & $9.66 \pm 1.07 \mathrm{a}$ & $9.91 \pm 1.26 \mathrm{a}$ & $9.98 \pm 1.28 \mathrm{a}$ & $8.68 \pm 1.41 b$ & $9.50 \pm 1.69 b$ & $10.05 \pm 2.11 \mathbf{a}$ \\
\hline MLW (cm plant $\left.{ }^{-1}\right)$ & $0.24 \pm 0.04 b$ & $0.31 \pm 0.06 \mathbf{a}$ & $0.31 \pm 0.08 \mathbf{a}$ & $0.28 \pm 0.10 \mathrm{~b}$ & $0.32 \pm 0.08 b$ & $0.39 \pm 0.07 \mathbf{a}$ \\
\hline LA $\left(\mathrm{cm}^{2}\right.$ plant $\left.^{-1}\right)$ & $0.76 \pm 0.15 b$ & $0.99 \pm 0.23 \mathbf{a}$ & $1.02 \pm 0.34 \mathbf{a}$ & $0.81 \pm 0.37 b$ & $1.01 \pm 0.39 \mathbf{a b}$ & $1.28 \pm 0.40 \mathbf{a}$ \\
\hline LRL (cm plant $\left.{ }^{-1}\right)$ & $6.32 \pm 1.30 b$ & $7.09 \pm 1.73 b$ & $8.68 \pm 2.68 \mathbf{a}$ & $8.77 \pm 3.07 \mathrm{a}$ & $7.87 \pm 1.70 \mathrm{a}$ & $7.56 \pm 2.32 \mathrm{a}$ \\
\hline $\mathrm{RN}\left(\right.$ plant $\left.^{-1}\right)$ & $13.07 \pm 3.63 \mathrm{a}$ & $15.14 \pm 2.98 \mathrm{ab}$ & $16.41 \pm 2.09 \mathrm{a}$ & $12.40 \pm 3.00 \mathrm{~b}$ & $16.93 \pm 3.84 a$ & $19.07 \pm 4.83 \mathrm{a}$ \\
\hline $\mathrm{RV}\left(\mathrm{cm} 3\right.$ plant $\left.^{-1}\right)$ & $0.08 \pm 0.01 b$ & $0.09 \pm 0.02 b$ & $0.14 \pm 0.07 a$ & $0.07 \pm 0.01 \mathrm{c}$ & $0.11 \pm 0.04 b$ & $0.15 \pm 0.08 \mathrm{a}$ \\
\hline $\operatorname{Co}(\mu \mathrm{S})$ & $217.7 \pm 17.01 b$ & $371.5 \pm 53.70 \mathbf{a}$ & $105.2 \pm 12.72 \mathrm{c}$ & $186.2 \pm 14.92 b$ & $426.1 \pm 134.1 \mathbf{a}$ & $56.09 \pm 40.04 c$ \\
\hline $\mathrm{SS}(\%)$ & $1.45 \pm 0.54 \mathbf{a}$ & $1.68 \pm 0.33 \mathbf{a}$ & $1.12 \pm 0.33 b$ & $1.51 \pm 0.32 \mathbf{a}$ & $1.79 \pm 0.51 \mathbf{a}$ & $1.08 \pm 0.31 b$ \\
\hline Pro $\left(\mu g^{-1}\right)$ & $5.72 \pm 2.56 \mathbf{a}$ & $2.48 \pm 1.49 b$ & $2.71 \pm 0.81 b$ & $5.78 \pm 2.81 \mathbf{a}$ & $1.86 \pm 0.49 b$ & $2.78 \pm 0.9 b$ \\
\hline TDM (g plant $\left.{ }^{-1}\right)$ & $37.17 \pm 5.83 c$ & $51.39 \pm 12.54 b$ & $70.63 \pm 26.44 \mathbf{a}$ & $41.5 \pm 11.55 b$ & $59.21 \pm 25.09 a$ & $74.39 \pm 26.76 a$ \\
\hline
\end{tabular}

Bars indicate $\mathrm{SD}(\mathrm{n}=3)$. Different letters indicate a significant difference $(P<0.05)$ between the stress durations. 


\section{The principal component analysis of rice seedling morphological and physiological traits}

The principal component analysis showed that RDM, SDM, SH, MLL, MLW, LA, $\mathrm{RN}$ and RV contributed to the first principal component (dehydration avoidance factor, DAF) (Table 5), while the LA was the major to the first principal component. ARI and RWC were summed up as the second principal component (comprehensive drought resistant factor, CDRF), RWC obviously supported on the factor. Co, SS and Pro were classified as the third principal component (drought tolerance factor, DTF), Co was leading component to the factor. In addition, RLR was called as fourth principal component (root induction factor, RIF).

Table 5. The principal component analysis of the root dry matter (RDM, $g$ plant $\left.{ }^{-1}\right)$, shoot dry matter (SDM, $g$ plant $\left.^{-1}\right)$, adversity resistance index (ARI), relative water content (RWC), seedling height (SH, cm plant $\left.{ }^{-1}\right)$, mean leaf length $\left(M L L, \mathrm{~cm} \mathrm{plant}^{-1}\right)$, mean leaf width (MLW, $\left.\mathrm{cm}_{\text {plant }}{ }^{-1}\right)$, leaf area $\left(L A, \mathrm{~cm}^{2}\right.$ plant $\left.^{-1}\right)$, longest root length $\left(L R L, \mathrm{~cm}_{\text {plant }}{ }^{-1}\right)$, root number $\left(R N\right.$, plant $\left.t^{-1}\right)$, root volume $\left(R V, \mathrm{~cm}^{3}\right.$ plant $\left.^{-1}\right)$, conductivity $(C o, \mu S)$, soluble sugar (SS, \%), proline (Pro, $\left.\mu \mathrm{g} \mathrm{g}^{-1}\right)$ and total dry matter $\left(\mathrm{g}\right.$ plant $\left.^{-1}\right)$ of drought stress intensity and stress duration under Yangliangyou 6 (YLY6) and Hanyou 113 (HY113)

\begin{tabular}{|c|c|c|c|c|c|c|c|c|c|c|c|c|c|c|}
\hline 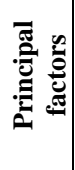 & 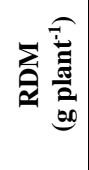 & 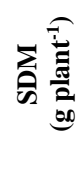 & $\frac{\bar{x}}{4}$ & 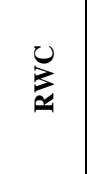 & क & 氙奇 & 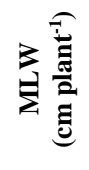 & 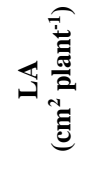 & 童 & 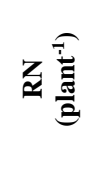 & 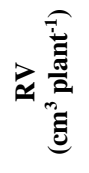 & $\begin{array}{l}\frac{\pi}{3} \\
0\end{array}$ & $\stackrel{0}{0}$ & 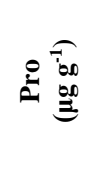 \\
\hline 1 & 0.846 & 0.936 & 0.516 & 0.354 & 0.886 & 0.839 & 0.883 & 0.947 & 0.51 & 0.751 & 0.856 & -0.576 & -0.55 & -0.62 \\
\hline 2 & -0.066 & -0.105 & 0.711 & 0.743 & 0.048 & 0.176 & -0.221 & -0.096 & 0.293 & -0.435 & 0.047 & 0.024 & 0.05 & 0.308 \\
\hline 3 & 0.358 & 0.072 & -0.028 & -0.035 & -0.113 & -0.067 & 0.014 & 0.007 & 0.457 & 0.125 & 0.249 & 0.647 & 0.598 & -0.623 \\
\hline 4 & -0.252 & -0.045 & 0.174 & 0.24 & 0.058 & 0.322 & 0.045 & 0.142 & -0.517 & 0.169 & -0.247 & 0.431 & 0.128 & -0.45 \\
\hline
\end{tabular}

\section{The relation and path analysis of principal component factors and dry matter accumulation}

Correlation analysis explored that (Fig. 4), the TDM significantly $(P<0.01)$ positively related to LA, RWC, Co and LRL. The LA also significantly $(P<0.01)$ negatively correlated with $\mathrm{Co}$, which indicated that dehydration avoidance factor, comprehensive drought resistant factor and drought tolerance factor related closely with biomass accumulation in rice seedling.

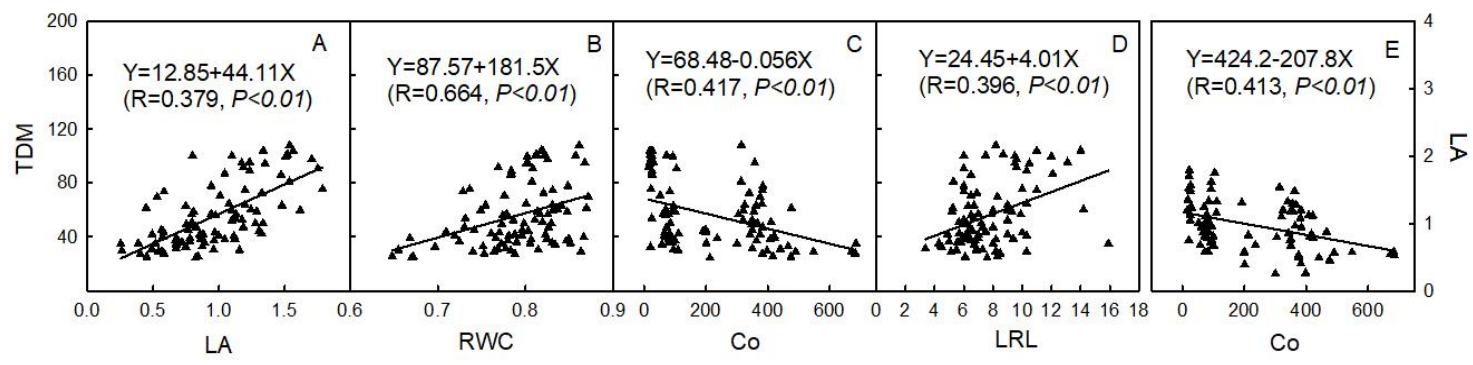

Figure 4. Relationship $(n=90)$ for total dry matter $\left(T D M, \mathrm{~g}_{\text {plant }}{ }^{-1}\right)$ with leaf area $\left(L A, \mathrm{~cm}^{2}\right.$ plant $\left.^{-1}\right)$, total dry matter (TDM, $g$ plant $\left.{ }^{-1}\right)$ with relative water content $(R W C)$, total dry matter (TDM, $g$ plant $\left.^{-1}\right)$ with conductivity $(C o, \mu S)$, total dry matter (TDM, $g$ plant $\left.{ }^{-1}\right)$ with longest root length (LRL, cm plant $\left.{ }^{-1}\right)$ and leaf area $\left(L A, \mathrm{~cm}^{2}\right.$ plant $\left.{ }^{-1}\right)$ with conductivity $(C o, \mu S)$ of stress intensity and stress duration under Yangliangyou 6 (YLY6) and Hanyou 113 (HY113) 
Path analysis (Table 6) showed that LA, RWC, Co and LRL had greater direct effect on TDM in rice seedling, respectively, and Co had good indirect effect on TDM through LA. It showed that drought dehydration factor, comprehensive drought resistant factor and drought tolerance factor had great separate effect on rice seedling growth.

Table 6. The path analysis $(n=90)$ for the direct or indirect effect on total dry matter (TDM, $g$ plant $\left.{ }^{-1}\right)$ by leaf area $\left(L A, \mathrm{~cm}^{2}\right.$ plant $\left.{ }^{-1}\right)$, relative water content $(R W C)$, conductivity $(C o, \mu S)$ and longest root length (LRL, cm plant $\left.{ }^{-1}\right)$ of drought stress intensity and duration under Yangliangyou 6 (YLY6) and Hanyou 113 (HY113)

\begin{tabular}{c|c|c|c|c}
\hline & \multicolumn{4}{|c}{ TDM (Y) } \\
\cline { 2 - 5 } & $\mathbf{X 1 \rightarrow Y}$ & $\mathbf{X 2} \rightarrow \mathbf{Y}$ & $\mathbf{X 3} \rightarrow \mathbf{Y}$ & $\mathbf{X 4} \rightarrow \mathbf{Y}$ \\
\hline LA (X1) & $\mathbf{0 . 5 3}$ & 0.06 & 0.03 & 0.05 \\
Co (X2) & $\mathbf{- 0 . 2 2}$ & $\mathbf{- 0 . 1 4}$ & -0.02 & -0.04 \\
RWC (X3) & 0.05 & 0.01 & $\mathbf{0 . 2 8}$ & 0.03 \\
LRL (X4) & 0.13 & 0.03 & 0.05 & $\mathbf{0 . 1 9}$ \\
\hline
\end{tabular}

\section{The association analysis of principal component factors and dry matter accumulation}

In two varieties, under 0\%-4 $\mathrm{d}$ (stress intensity (SI)-stress duration (SD), 15\%-4 d, 10\%-4 d and 20\%-4 d condition (Table 7), RWC as the key enabling factor on TDM, followed by LA significantly contributed to TDM under 0\%-8 d, 10\%-8 d, 10\%-12 d, 20\%-12 d and 25\%-12 d condition. Co mainly supported TDM in 0\%-8 d, 10\%-8 d, 20\%-8 d, 20\%-12 d and 25\%-8 d; Co lowered contribution to rice seedling in 10\%-4 d, $15 \%-4 \mathrm{~d}, 20 \%-4 \mathrm{~d}$ and $25 \%-4 \mathrm{~d}$. LRL was the main factor for TDM in 10\%-4 $\mathrm{d}$ and $15 \%-8 \mathrm{~d}$ condition.

Table 7. The association analysis of the leaf area $\left(L A, \mathrm{~cm}^{2}\right.$ plant $\left.{ }^{-1}\right)$, relative water content $(R W C)$, conductivity $(C o, \mu S)$ and longest root length $\left(L R L, \mathrm{~cm}_{\text {plant }}{ }^{-1}\right)$ with total dry matter $\left(T D M, g\right.$ plant $\left.^{-1}\right)$ of stress intensity (SI) and stress duration (ST) under Yangliangyou 6 (YLY6) and Hanyou 113 (HY113)

\begin{tabular}{c|c|c|c|c|c|c|c|c|c}
\hline \multirow{2}{*}{$\begin{array}{c}\text { Stress } \\
\text { intensity (\%) }\end{array}$} & $\begin{array}{c}\text { Stress } \\
\text { duration (d) }\end{array}$ & \multicolumn{6}{|c|}{ YLY6 } & \multicolumn{5}{c}{ HY113 } \\
\hline \multirow{3}{*}{0} & 4 & 0.46 & $\mathbf{1 . 0 0}$ & 0.58 & 0.48 & 0.67 & $\mathbf{0 . 8 7}$ & $\mathbf{0 . 8 1}$ & $\mathbf{0 . 5 4}$ \\
& 8 & $\mathbf{0 . 9 9}$ & 0.65 & $\mathbf{0 . 6 8}$ & $\mathbf{0 . 5 1}$ & $\mathbf{0 . 6 8}$ & 0.58 & 0.67 & 0.46 \\
& 12 & 0.58 & 0.59 & 0.62 & 0.47 & 0.50 & 0.51 & 0.64 & 0.46 \\
\hline \multirow{3}{*}{10} & 4 & 0.50 & 0.56 & 0.53 & 0.61 & $\mathbf{0 . 8 9}$ & $\mathbf{0 . 9 8}$ & 0.64 & $\mathbf{0 . 6 1}$ \\
& 8 & 0.60 & 0.52 & 0.62 & 0.58 & 0.53 & 0.90 & $\mathbf{0 . 6 8}$ & 0.48 \\
& 12 & $\mathbf{0 . 8 8}$ & $\mathbf{0 . 9 1}$ & $\mathbf{0 . 6 3}$ & $\mathbf{0 . 9 8}$ & 0.56 & 0.57 & $\mathbf{0 . 6 8}$ & 0.55 \\
\hline \multirow{3}{*}{15} & 4 & 0.50 & 0.72 & 0.48 & $\mathbf{0 . 6 5}$ & 0.49 & $\mathbf{0 . 9 3}$ & 0.62 & 0.54 \\
& 8 & 0.46 & $\mathbf{0 . 9 6}$ & $\mathbf{0 . 6 8}$ & 0.56 & 0.46 & 0.54 & $\mathbf{0 . 6 6}$ & $\mathbf{0 . 6 6}$ \\
& 12 & $\mathbf{0 . 4 7}$ & $\mathbf{0 . 9 4}$ & 0.64 & 0.48 & $\mathbf{0 . 5 0}$ & 0.46 & 0.64 & 0.45 \\
\hline \multirow{3}{*}{20} & 4 & 0.68 & $\mathbf{0 . 9 5}$ & 0.51 & 0.46 & $\mathbf{0 . 5 6}$ & $\mathbf{0 . 8 7}$ & 0.46 & 0.50 \\
& 8 & 0.46 & 0.45 & $\mathbf{0 . 6 8}$ & 0.46 & 0.50 & 0.46 & $\mathbf{0 . 6 8}$ & $\mathbf{0 . 5 2}$ \\
& 12 & $\mathbf{0 . 7 1}$ & 0.24 & 0.35 & $\mathbf{0 . 7 0}$ & 0.46 & 0.51 & $\mathbf{0 . 6 8}$ & 0.45 \\
\hline \multirow{3}{*}{25} & 4 & 0.58 & $\mathbf{0 . 9 3}$ & 0.49 & 0.54 & 0.53 & $\mathbf{0 . 6 7}$ & 0.63 & $\mathbf{0 . 9 5}$ \\
& 8 & 0.52 & 0.64 & 0.62 & 0.52 & 0.46 & 0.47 & $\mathbf{0 . 6 7}$ & 0.48 \\
& 12 & $\mathbf{0 . 6 8}$ & 0.61 & $\mathbf{0 . 6 7}$ & $\mathbf{0 . 5 8}$ & $\mathbf{0 . 5 7}$ & 0.52 & 0.60 & 0.55 \\
\hline
\end{tabular}


It is suggested that comprehensive drought resistant factor primarily supported seedling growth at 8-12 d stress duration at 10-15\% PEG solution; under the coupling of the 20-25\% PEG concentration and $12 \mathrm{~d}$ stress duration condition, drought dehydration factor mainly supported the seedling growth. Drought tolerance factor had an imperative role in seedling growth at 8-12 d stress duration under 15-20\% PEG solution. The root induction factor was the major factor relating to seedling growth under $4 \mathrm{~d}$ drought stress duration with 10-15\% PEG solution.

\section{Discussion}

Drought stress intensity had a great contribution to rice seedling growth compared with drought processing duration

Drought stress intensity and duration significantly alters rice seedling growth. In the present study, rice seedling growth showed a reducing trend in prolonged drought stress duration, while mild and moderate drought stress intensity (10-15\% PEG concentration) enhanced rice seedling growth for all drought duration. In addition, shoot and root growth of rice seedling were more sensitive to drought stress intensity compared with drought duration, and root growth of rice seedling had a lower tolerance to drought stress intensity compared with the shoot of rice seedling.

Crop seedling growth slightly reduced with extending drought duration compared with control (Asai et al., 2009; Duan et al., 2017). As drought stress intensity increases, the root growth of seedling was decreased. Conversely, increases root physiological activity (root vigor and protective enzyme) (Sharma and Dubey, 2005; Ding et al., 2016). Rice shoot growth (biomass accumulation) was obviously reduced by lower leaf productivity (changed leaf morphology and gas exchange) under severe drought stress condition ( $\mathrm{Lu}$ and Neumann, 1999). While moderate drought stress supported rice seedling growth because which motivated the compensatory capacity of the seedling (Sharma and Dubey, 2005; Yu et al., 2016). These data found that root and shoot of crop seedling were sensitive to drought stress while moderate drought stress had a positive effect on rice seedling growth.

In the present research, drought duration had less threaten to rice seedling growth compared to drought stress intensity, because rice seedling during long-term drought stress could counteract the adverse impact of moderate drought stress on rice seedling growth, or the compensatory capacity of rice seedling could be stimulated by long-term drought duration. Compared with the aerial part of rice seedling, root had a lower tolerance capacity to drought stress due to more sensitive to drought stress during rice seedling and was a source of drought stress signal. Thus, long-term drought duration or mild or moderate drought stress intensity had less effect on rice seedling growth. And to maintain root vigor was more benefit for rice seedling growth.

Drought stress intensity and processing duration could impact on drought dehydration factor, comprehensive drought resistant factor, drought tolerance factor, root induction factor and the relationship among them

The impact of drought stress on rice seedling growth and development was not only related to the drought stress intensity and processing duration, but also corrected to drought resistance (drought dehydration and drought tolerance) of crops (Liu et al., 2003; Özdemir et al., 2004; Farooq et al., 2009). In the present research, drought 
dehydration, drought tolerance, comprehensive drought resistant and root induction factor increased when the plants were exposed to extending drought duration; moreover, drought dehydration, drought tolerance and comprehensive drought factor had greater value at moderate drought stress (15\% PEG concentration) intensity within long term drought duration, while optimal root induction factor appeared at mild drought stress (10\% PEG concentration) during medium and long term drought duration. And drought dehydration, comprehensive drought resistant, drought tolerance and root induction factors within each drought stress intensity, duration or their combination had a great independent effect.

Crops simultaneously had drought dehydration and tolerance ability (Liu et al., 2003; Chaves et al., 2003; Chang, 2008; Farooq et al., 2009). Drought tolerance or dehydration in crops showed a different size when crops faced a different drought stress intensity (Yu et al., 2016; Duan et al., 2017). Drought dehydration and drought tolerate ability had different ways to maintain the crops growth and avoid the much number water lose: short-term drought stress duration inspired the drought tolerance ability, while long-term drought stress duration easily induced the drought resistance (Yue et al., 2006; Farooq et al., 2009; Li et al., 2012). And these factors were respectively, controlled by a set of independent genetic characteristics, such as, root distribution (the longest root length) in the soil layer and leaf area was adjusted by related gene (Zhang et al., 2001; Yue et al., 2006; Du et al., 2018). In addition, under the assist of the compensation ability, mild and moderate drought stress intensity stimulated the formation of protection mechanisms in tissue cells to avoid the injury of tissue cells (Asai et al., 2009; Duan et al., 2017). With the raising stress duration, the generation of new cells replaced or eliminated which destroyed tissue cells or other matter in cells (Ming and Wang, 2002; Kottapalli et al., 2012). And the great activity or function of the new tissue cells raised the drought resistance ability supporting the rice seedling growth. Thus, drought dehydration played a crucial role in drought stress duration, while drought tolerance had an obvious response to drought stress intensity to support rice seedling growth. In addition, less alteration of seedling morphological traits, when seedling accumulated higher Pro and SS content, and rice seedling had low assimilate productivity during seedling stage. This indicated that the accumulated assimilates in seedling provided energy for the formation of the proteins and enzymes related to drought tolerance, while reducing the energy to support the morphogenesis of rice seedling, so that drought dehydration factor and drought tolerance factor showed an independent effect on rice seedling growth. And these factors had different acting time during drought stress.

\section{Drought stress intensity and duration impacted on drought dehydration factor, comprehensive drought resistant factor, drought tolerance factor and root induction factor to adjust the rice seeding growth}

In the present study, the rice seedling maintained the growth during drought stress, mainly supported by greater root induction, drought tolerance and drought dehydration factor at short-term, medium-term or long-term duration under mild drought stress intensity, respectively. The comprehensive drought resistant factor among all drought duration in moderate drought stress intensity, drought dehydration factor and drought tolerance factor at long-term processing time in severe stress intensity respectively played a crucial role in rice seedling growth. 
The contribution of drought tolerance factor on rice seedling growth was mainly great physiological activity (polyphenols, flavonoids and antioxidant activity) under severe drought stress (Hussain et al., 2016, 2017). However, the formation of protective enzyme or cell activity was induced by environmental stress and reducing with increasing drought stress intensity (Manivannan et al., 2007; Samota et al., 2017). While Drought dehydration played a crucial role in long-term drought stress (Farooq et al., 2009; Li et al., 2012; Hussain et al., 2017). About RWC (comprehensive drought resistant factor) was considered to evaluate the response-ability of plant to drought stress (Farooq et al., 2009; Anjum et al., 2011), and the comprehensive drought resistant factor was supported by root or shoot physiological activity, decreasing water loss and enhancing physiological water use efficiency in rice seedling (Kaydan et al., 2008), but the comprehensive drought resistant factor also reduced with raising drought intensity (Nayyar et al., 2006; Anjum et al., 2011). Root induction factor including root distribution and the growth rate of root, rice root distribution in deep soil layer significantly related with drought stress and root growth rate was used to evaluate adaptability to stress environment, root growth and physiological activity (synthesis of protein) were stimulated by mild and moderate drought stress to increase water absorption (Farooq et al., 2009; Pérez et al., 2010). We concluded that drought tolerance factor and the comprehensive drought resistant factor reduced within severe drought stress, while root induction factor raised in mild and moderate drought stress, drought dehydration played a crucial role in long term drought stress. A greater drought tolerance showed under short-term drought stress; moreover, drought tolerance factor mainly improving drought resistance ability, the comprehensive drought resistant factor mainly raising the water use ability and root induction factor mainly promoting water absorption which all supported drought resistance ability during drought stress.

In the present study, the root is the first organ to contact with drought stress and is more sensitive to drought stress. This showed that root had an obvious change within a short time to face drought stress intensity. This improved root water absorption to support rice seedling growth in the short-term stress. Drought tolerance factor plays a key role in stress environment and mild drought stress had less effect on seedling growth. While mid drought stress coupled with medium-term drought duration could stimulate the drought tolerance factor by improving compensation ability to promote the drought tolerance ability of rice seedling. Under moderate drought stress, comprehensive drought resistant factor did not show a declining trend, it could be caused by great effect of the protection mechanism and drought dehydration factor, in each drought duration, respectively. This further directly supported rice seedling growth.

The drought dehydration factor and drought tolerance factor of rice seedling respectively including shoot or root morphological and physiologic traits. Drought dehydration factor and drought tolerance factor could take advantage during long term drought duration with severe drought stress, because the accumulation of carbohydrates, soluble proteins, some osmotic substances and the protective enzyme during a relatively long time improved water absorption or storage avoiding the destruction of free radicals to cells supporting rice seedling growth during severe drought stress. In addition, drought dehydration factor was supported by morphology of rice seedlings, such as root and leaf, which was the foundation of matter and energy to response or relieve drought stress of rice seedling. Hence, drought dehydration factor had a great contribution to rice seedling growth during a long-term drought stress condition. 


\section{Conclusion}

Rice seedling growth in different drought stress intensity and duration was associated with their different stress resistant mode, including drought dehydration avoidance factor, comprehensive drought resistant factor, drought tolerance factor and root induction factor. Moreover, these factors have strong independent effect on seedling growth during different drought stress intensity and duration. The improved rice seedling growth was mainly associated with greater root induction factor, drought tolerance and drought dehydration factor at short, medium or long-term duration under mild drought stress intensity, respectively. While drought dehydration and drought tolerance factor at long-term processing duration under severe stress intensity played a crucial role to support the rice seedling growth. Thus, to focus on drought resistant traits under different drought environment are important way to evaluate rice seedling growth in rice dry cultivation systems.

At present, the breeding of drought-resistance rice variety uses the genetic advantage of rice varieties. In the future, genome, transcriptome, proteome, or metabolomics can be applied to study the interaction mechanism between the drought resistance ability (drought resistance, drought avoidance, drought dehydration and drought tolerance) and the environment (drought stress). It is of great significance to the future development of rice dry cultivation (reduce energy-saving production).

Acknowledgements. This study was funded by the National Natural Science Foundation of China (31801291) and State Key Special Program (2017YFD0301400).

Conflict of interests. There is no conflict of interests to declare.

\section{REFERENCES}

[1] Anjum, S. A., Xie, X. Y., Wang, L. C., Saleem, M. F., Man, C., Lei, W. (2011): Morphological, physiological and biochemical responses of plants to drought stress. African Journal of Agricultural Research 6: 2026-2032.

[2] Asai, H., Samson, B. K., Stephan, H. M., Songyikhangsuthor, K., Homma, K., Kiyono, Y. (2009): Biochar amendment techniques for upland rice production in northern Laos: 1. soil physical properties, leaf spad and grain yield. - Field Crops Research 111: 81-84.

[3] Bandurska, H., Stroiński, A., Kubiś, J. (2003): The effect of jasmonic acid on the accumulation of aba, proline and spermidine and its influence on membrane injury under water deficit in two barley genotypes. - Acta Physiologiae Plantarum 25: 279-285.

[4] Basu, S., Roychoudhury, A., Saha, P. P., Sengupta, D. N. (2010): Differential antioxidative responses of indica rice cultivars to drought stress. - Plant Growth Regulation 60: 51-59.

[5] Bates, L. S., Waldren, R. P., Teare, I. D. (1973): Rapid determination of free proline for water stress studies. - Plant and Soil 39: 205-207.

[6] Chang, X. (2008): Study advances on the physiological adaptation mechanism to drought stress by plant. - Journal of Anhui Agricultural Science 36: 7549-7551.

[7] Chaves, M. M., Maroco, J. P., Pereira, J. S. (2003): Understanding plant responses to drought - from genes to the whole plant. - Functional Plant Biology 30: 239-264.

[8] Chen, K., Arora, R. (2011): Dynamics of the antioxidant system during seed osmopriming, post-priming germination, and seedling establishment in spinach (Spinacia oleracea L.). - Plant Science 180: 212-20. 
[9] Ding, L., Li, Y., Wang, Y., Gao, L., Wang, M., Chaumont, F., Shen, Q., Guo, S. (2016): Root $\mathrm{ABA}$ accumulation enhances rice seedling drought tolerance under ammonium supply: Interaction with aquaporins. - Frontier in Plant Science 7: 1206.

[10] Du, H., Huang, F., Wu, N., Li, X., Hu, H., Xiong, L. (2018): Integrative regulation of drought escape through ABA dependent and independent pathways in rice. - Molecular Plant. DOI: 10.1016/j.molp.2018.01.004.

[11] Duan, S., Yang, A., Huang, Y. (2017): Effects of water stress on growth and physiological features and yield of rice. - China Rice 23: 36-42.

[12] Farooq, M., Wahid, A., Kobayashi, N. (2009): Plant drought stress: effects, mechanisms and management. - Agronomy for Sustainable Development 29: 185-212.

[13] Huang, M., Zou, Y. B., Jiang, P. (2011): Relationship between grain yield and yield components in super hybrid rice. - Agricultural Sciences in China 10: 1537-1544.

[14] Hussain, M., Farooq, M., Lee, D. J. (2017): Evaluating the role of seed priming in improving drought tolerance of pigmented and non-pigmented rice. - Crop Science 203: 269-276.

[15] Hussain, S., Khan, F., Hussain, H. A., Nie, L. (2016): Physiological and biochemical mechanisms of seed priming-induced chilling tolerance in rice cultivars. - Frontier in Plant Science 7: 116.

[16] Kaydan, D., Yagmur, M. (2008): Germination, seedling growth and relative water content of shoot in different seed sizes of triticale under osmotic stress of water and $\mathrm{NaCl}$. African Journal of Biotechnology 7: 2862-2868.

[17] Kottapalli, K. R., Kottapalli, P., Payton, P. (2012): Peanut Seed Development: Molecular Mechanisms of Storage Reserve Mobilization and Effect of Water Deficit Stress on Seed Metabolism. - In: Agrawal, G. K., Rakwal, R. (eds.) Seed Development: OMICS Technologies Toward Improvement of Seed Quality and Crop Yield. - Springer, Netherlands, pp: 143-169.

[18] Kusaka, M., Ohta, M., Fujimura, T. (2005): Contribution of inorganic components to osmotic adjustment and leaf folding for drought tolerance in pearl millet. - Physiology Plantarum 125: 474-489.

[19] Li, H. S. (2012): Modern Plant Physiology. - Higher Education Press, Beijing, pp: $352-$ 357.

[20] Li, J., Besseau, S., Törönen, P., Sipari, N., Kollist, H., Holm, L. (2013): Defense-related transcription factors wrky70 and wrky54 modulate osmotic stress tolerance by regulating stomatal aperture in arabidopsis. - New Phytologist 200: 457-72.

[21] Liu, C. L., Chen, H. P., Liu, E. E., Peng, X. X., Lu, S. Y., Guo, Z. F. (2003): Multiple tolerance of rice to abiotic stresses and its relationship with aba accumulation. - Acta Agronomica Sinica 29: 725-729.

[22] Lu, Z., Neumann, P. M. (1999): Water stress inhibits hydraulic conductance and leaf growth in rice seedlings but not the transport of water via mercury-sensitive water channels in the root. - Plant Physiology 120: 143-152.

[23] Luo, L. J. (2010): Breeding for water-saving and drought-resistance rice (WDR) in China. - Journal of Experimental Botany 61: 3509.

[24] Manivannan, P., Abdul, J. C., Kishorekumar, A., Sankar, B., Somasundaram, R., Sridharan, R. (2007): Changes in antioxidant metabolism of Vigna unguiculata, (L.) walp. by propiconazole under water deficit stress. - Colloids and Surfaces B Biointerfaces 57: 69-74.

[25] Ming, L., Wang, G. (2002): Effect of drought stress on activities of cell defense enzymes and lipid peroxidation in glycyrrhiza uralensis seedings. - Acta Ecologica Sinica 22: 503507.

[26] Nayyar, H., Gupta, D. (2006): Differential sensitivity of $\mathrm{C}_{3}$, and $\mathrm{C}_{4}$, plants to water deficit stress: association with oxidative stress and antioxidants. - Environmental and Experimental Botany 58: 106-113. 
[27] Nazarli, H., Ahmadi, A., Hadian, J. (2014): Salicylic acid and methyl jasmonate enhance drought tolerance in chamomile plants. - Journal of HerbMed Pharmacology 3: 87-92.

[28] Özdemir, F., Bor, M., Demiral, T. (2004): Effects of 24-epibrassinolide on seed germination, seedling growth, lipid peroxidation, proline content and antioxidative system of rice (Oryza sativa L.) under salinity stress. - Plant Growth Regulation 42: 203211.

[29] Pérez, M. B. E., Gidekel, M., Segura, N. M., Herrera, E. L., Ochoa, A. N. (2010): Effects of water stress on plant growth and root proteins in three cultivars of rice (Oryza sativa L.) with different level of drought tolerance. - Physiology Plantarum 96: 284-290.

[30] Pirdashti, H., Sarvestani, Z. T., Nematzadeh, G., Ismail, A. (2003): Effect of water stress on seed germination and seedling growth of rice (Oryza sativa L.) genotypes. - Journal of Agronomy 6: 217-222.

[31] Samota, M. K., Sasi, M., Awana, M., Yadav, O. P., Mithra, S. V. A., Tyagi, A. (2017): Elicitor-induced biochemical and molecular manifestations to improve drought tolerance in rice (Oryza sativa L.) through seed-priming. - Frontier in Plant Science 8: 934.

[32] Sharma, P., Dubey, R. S. (2005): Drought induces oxidative stress and enhances the activities of antioxidant enzymes in growing rice seedlings. - Plant Growth Regulation 46: 209-221.

[33] Sun, Y., Ma, J., Sun, Y., Xu, H., Yang, Z., Liu, S. (2012): The effects of different water and nitrogen managements on yield and nitrogen use efficiency in hybrid rice of China. Field Crops Research 127: 85-98.

[34] Technology and Information Center of China Rice Research Institute, National Rice Data Center (2016): Technology Wikipedia Encyclopedia of Upland Rice. www.ricedata.cn/Baike/2016, 3-10.

[35] Wang, S., Hu, Y., She, K. (2007): Gray relational grade analysis of agronomical and physi-biochemical traits related to drought tolerance in wheat. - Scientia Agricultura Sinica 40: 2452-2459.

[36] Xu, Y. Z., Wu, W. G., Chen, G., Ding, G. L. (2017): Study and integration of key techniques for high-yielding and water-saving cultivation of upland hybrid rice. - China Rice 23: 39-43.

[37] Yang, J. C., Yong, D. U., Liu, H. (2008): Cultivation approaches and techniques for annual super-high-yielding of rice and wheat in the lower reaches of Yangzi River. Scientia Agricultura Sinica 41: 1611-1621.

[38] Ye, Y., Liang, X., Chen, Y., Liu, J., Gu, J., Guo, R., Li, L. (2013): Alternate wetting and drying irrigation and controlled-release nitrogen fertilizer in late-season rice. Effects on dry matter accumulation, yield, water and nitrogen use. - Field Crops Research 144: 212224.

[39] Yu, X., James, A. T., Yang, A., Jones, A. (2016): A comparative proteomic study of drought-tolerant and drought-sensitive soybean seedlings under drought stress. - Crop Pasture Science 67: 528-540.

[40] Yue, B., Xue, W., Xiong, L., Yu, X., Luo, L., Cui, K. (2006): Genetic basis of drought resistance at reproductive stage in rice: separation of drought tolerance from drought avoidance. - Genetics 172: 1213.

[41] Zhang, J., Zheng, H. G., Aarti, A., Pantuwan, G., Nguyen, T. T., Tripathy, J. N. (2001): Locating genomic regions associated with components of drought resistance in rice: comparative mapping within and across species. - Theoretical and Applied Genetics 103: 19-29.

[42] Zhang, M., Jin, Z. Q., Zhao, J., Zhang, G., Wu, F. (2015): Physiological and biochemical responses to drought stress in cultivated and Tibetan wild barley. - Plant Growth Regulation 75: 567-574.

[43] Zhang, Z., Qu, W. (2003): Guidance of plant Physiology Experiments. 3rd Ed. - Higher Education Press, Beijing, pp. 127-132. 
[44] Zheng, M., Ye, T., Hussain, S., Jiang, Q., Peng, S., Huang, J. (2016): Seed priming in dry direct-seeded rice: consequences for emergence, seedling growth and associated metabolic events under drought stress. - Plant Growth Regulation 78: 167-178. 